\title{
INTEGRALS OF BESSEL FUNCTIONS
}

\author{
D. BABUSCI, G. DATTOLI, B. GERMANO, M. R. MARTINELLI, AND P. E. RICCI
}

Abstract. We use the operator method to evaluate a class of integrals involving Bessel or Besseltype functions. The technique we propose is based on the formal reduction of these family of functions to Gaussians.

Integrals of the type

$$
I_{n}(a, b, \alpha)=\int_{-\infty}^{\infty} \mathrm{d} x(a x+b)^{n} \mathrm{e}^{-\alpha x^{2}}
$$

can be calculated using a general procedure based on the method of generating function (GF) [3] Multiplying both sides of eq. (10) by $t^{n} / n$ ! and summing up over $n$, we find

$$
\begin{aligned}
G(a, b, \alpha) & =\sum_{n=0}^{\infty} \frac{t^{n}}{n !} I_{n}(\alpha, \beta)=\mathrm{e}^{b t} \int_{-\infty}^{\infty} \mathrm{d} x \mathrm{e}^{-\alpha x^{2}+t a x} \\
& =\sqrt{\frac{\pi}{\alpha}} \exp \left(\frac{t^{2} a^{2}}{4 \alpha}+b t\right) .
\end{aligned}
$$

By exploiting the GF of the two-variable Hermite polynomials [2]

$$
\sum_{n=0}^{\infty} \frac{t^{n}}{n !} H_{n}(x, y)=\mathrm{e}^{x t+y t^{2}} \quad H_{n}(x, y)=n ! \sum_{k=0}^{[n / 2]} \frac{x^{n-2 k} y^{k}}{(n-2 k) ! k !},
$$

from eq. (2) we get

$$
G(a, b, \alpha)=\sqrt{\frac{\pi}{\alpha}} \sum_{n=0}^{\infty} \frac{t^{n}}{n !} H_{n}\left(b, \frac{a^{2}}{4 \alpha}\right)
$$

and, thus

$$
I_{n}(a, b, \alpha)=\sqrt{\frac{\pi}{\alpha}} H_{n}\left(b, \frac{a^{2}}{4 \alpha}\right) .
$$

In a recent series of papers [3] it has been proved that the Bessel functions can be formally reduced to Gaussians according to the following representation

$$
J_{\nu}(x)=\left(\frac{x \hat{c}}{2}\right)^{\nu} \exp \left\{-\hat{c}\left(\frac{x}{2}\right)^{2}\right\} \varphi(0)
$$

where

$$
\hat{c}^{\mu} \varphi(0)=\varphi(\mu), \quad \varphi(\mu)=\frac{1}{\Gamma(\mu+1)} .
$$

The properties of the operator $\hat{c}$ have been extensively discussed in [3]. By treating it as an ordinary constant, we find, for example,

$$
B_{0}(\alpha)=\int_{-\infty}^{\infty} \mathrm{d} x J_{0}(\sqrt{\alpha} x)=\frac{2}{\sqrt{\alpha}}
$$

2000 Mathematics Subject Classification. Primary 33.

Key words and phrases. Integrals, generating function method, Bessel-type functions. 
and, in analogy with eq. (1), for $\nu>n(\nu \in \mathbb{R})$

$$
\begin{aligned}
\int_{-\infty}^{\infty} \mathrm{d} x J_{\nu}(\sqrt{\alpha} x) \frac{(a x+b)^{n}}{(\sqrt{\alpha} x)^{\nu}} & =\frac{1}{2^{\nu-1}} \frac{\sqrt{\pi}}{\sqrt{\alpha}} \hat{c}^{\nu-1 / 2} H_{n}\left(b, \frac{a^{2}}{\alpha \hat{c}}\right) \\
& =\frac{1}{2^{\nu-1}} \frac{\sqrt{\pi}}{\sqrt{\alpha}} B_{n}\left(b, \frac{a^{2}}{\alpha} ; \nu\right)
\end{aligned}
$$

where we have introduced the polynomials

$$
B_{n}(x, y ; \nu)=n ! \sum_{k=0}^{[n / 2]} \frac{x^{n-2 k} y^{k}}{(n-2 k) ! k ! \Gamma\left(\nu-k+\frac{1}{2}\right)},
$$

whose properties will be briefly described later. As a consequence of this result, for any function $f(x)$ that can be written as

$$
f(x, m)=\sum_{k=0}^{m} f_{k} x^{k} \quad(m<\nu),
$$

the following identify holds

$$
\int_{-\infty}^{\infty} \mathrm{d} x J_{\nu}(\sqrt{\alpha} x) \frac{f(a x+b)}{(\sqrt{\alpha} x)^{\nu}}=\frac{1}{2^{\nu-1}} \frac{\sqrt{\pi}}{\sqrt{\alpha}} \sum_{k=0}^{m} f_{k} B_{k}\left(b, \frac{a^{2}}{\alpha} ; \nu\right) .
$$

It is interesting to note that the linear combination of Bessel functions

$$
F_{n}(x ; a, b)=\sum_{k=0}^{n}\left(\begin{array}{l}
n \\
k
\end{array}\right) a^{n-k} b^{k} J_{n-k}(x)
$$

can be written as

$$
F_{n}(x ; a, b)=\left(\frac{a x}{2} \hat{c}+b\right)^{n} \exp \left\{-\hat{c}\left(\frac{x}{2}\right)^{2}\right\} \varphi(0)
$$

and, therefore, according to eq. (4), the following identity holds

$$
\begin{aligned}
\int_{-\infty}^{\infty} \mathrm{d} x F_{n}(x ; a, b) & =2 \sqrt{\pi} \hat{c}^{-1 / 2} H_{n}\left(b, \frac{a^{2}}{4} \hat{c}\right) \varphi(0) \\
& =2 \sqrt{\pi} n ! \sum_{k=0}^{[n / 2]} \frac{b^{n-2 k} a^{2 k}}{4^{k}(n-2 k) ! k ! \Gamma\left(k+\frac{1}{2}\right)} .
\end{aligned}
$$

The method illustrated is more general than it may appear and can indeed be extended to other families of Bessel-like functions. For example, the spherical Bessel functions [5]

$$
j_{n}(x)=\sqrt{\frac{\pi}{2 x}} J_{n+1 / 2}(x)
$$

within the present formalism can be written as

$$
j_{n}(x)=\frac{\sqrt{\pi}}{2^{n+1}} x^{n} \hat{c}^{n+1 / 2} \exp \left\{-\hat{c}\left(\frac{x}{2}\right)^{2}\right\} \varphi(0) .
$$

By using this expression, the integral

$$
b_{n}=\int_{-\infty}^{\infty} \mathrm{d} x j_{n}(x),
$$

can easily be calculated by exploiting the GF method, getting

$$
b_{2 n}=\frac{\pi}{2^{2 n}} \frac{(2 n) !}{(n !)^{2}}, \quad b_{2 n+1}=0 .
$$


The Struve functions are defined by the series [5]

$$
\mathbf{H}_{\nu}(x)=\sum_{k=0}^{\infty} \frac{(-1)^{k}}{\Gamma\left(k+\frac{3}{2}\right) \Gamma\left(k+\nu+\frac{3}{2}\right)}\left(\frac{x}{2}\right)^{2 k+\nu+1}
$$

Albeit in a slightly different form, even in this case we can apply the operational method, and this functions can be written as

$$
\mathbf{H}_{\nu}(x)=\hat{c}_{1}^{1 / 2} \hat{c}_{2}^{\nu+1 / 2}\left(\frac{x}{2}\right)^{\nu+1} \frac{1}{1+\hat{c}_{1} \hat{c}_{2}\left(\frac{x}{2}\right)^{2}} \varphi_{1}(0) \varphi_{2}(0)
$$

where the operator $\hat{c}_{i},(i=1,2)$ acts only on $\varphi_{i}$ and verifies the identity (6). Moreover, the use of the Laplace allows us to write

$$
\mathbf{H}_{\nu}(x)=\hat{c}_{1}^{1 / 2} \hat{c}_{2}^{\nu+1 / 2}\left(\frac{x}{2}\right)^{\nu+1} \int_{0}^{\infty} \mathrm{d} s \exp \left\{-s\left[1+\hat{c}_{1} \hat{c}_{2}\left(\frac{x}{2}\right)^{2}\right]\right\} \varphi_{1}(0) \varphi_{2}(0)
$$

that can be used to prove that, for $\mu+\nu$ not an even integer, the following identity holds

$$
\int_{0}^{\infty} \mathrm{d} x x^{\mu} \mathbf{H}_{\nu}(x)=(-1)^{\mu+\nu} \frac{2^{\mu} \pi}{\sin \left[(\mu+\nu) \frac{\pi}{2}\right]} \frac{1}{\Gamma\left(\frac{1-\mu-\nu}{2}\right) \Gamma\left(\frac{1-\mu+\nu}{2}\right)} .
$$

As a final example, we consider the Wright-Bessel functions [4]

$$
W_{\alpha, \beta}(x)=\sum_{k=0}^{\infty} \frac{x^{k}}{k ! \Gamma(k \alpha+\beta)}
$$

$(\beta \notin \mathbb{Z}$ when $\alpha<0)$ that can formally be defined as

$$
W_{\alpha, \beta}(x)=\hat{c}^{\beta-1} \exp \left(\hat{c}^{\alpha} x\right) \varphi(0) .
$$

By using this expression it's easy to show that

$$
\int_{-\infty}^{\infty} \mathrm{d} x W_{\alpha, \beta}\left(-x^{2}\right)=\sqrt{\pi} \frac{1}{\Gamma(\beta-\alpha / 2)},
$$

and $(d>0)$

$$
\int_{0}^{\infty} \mathrm{d} x W_{\alpha, \beta}(-x) \mathrm{e}^{-d x}=\frac{1}{d} E_{\alpha, \beta}\left(-\frac{1}{d}\right)
$$

where

$$
E_{\alpha, \beta}(x)=\sum_{k=0}^{\infty} \frac{x^{k}}{\Gamma(\alpha k+\beta)}
$$

is the modified Mittag-Leffler function [4].

Now, let us coming back to the polynomials $B_{n}(x, y ; \nu)$, introduced in eq. (9). These are auxiliary Hermite-like polynomials, and their properties can be deduced from those of Hermite polynomials written in umbral form. For example, by using the first of eq. (3) and eq. (24), it can be shown that the generating function is given by

$$
\sum_{n=0}^{\infty} \frac{t^{n}}{n !} B_{n}(x, y ; \nu)=\mathrm{e}^{x t} W_{-1, \nu+1 / 2}\left(y t^{2}\right)
$$

It is also interesting to note that these polynomials satisfy the differential equation

$$
\hat{D}_{y} B_{n}(x, y ; \nu)=\partial_{x}^{2} B_{n}(x, y ; \nu) \quad B_{3}(x, 0 ; \nu)=x^{n} \varphi\left(\nu-\frac{1}{2}\right)
$$


where the derivative operator $\hat{D}_{y}=\hat{c} \partial_{y}$ has been introduced. Therefore, we get

$$
B_{n}(x, y ; \nu)=\exp \left(\hat{c}^{-1} y \partial_{x}^{2}\right) x^{n} \varphi\left(\nu-\frac{1}{2}\right),
$$

that, taking into account the operational definition of the Hermite polynomials

$$
H_{n}(x, y)=\exp \left(y \partial_{x}^{2}\right) x^{n},
$$

can be easily shown to coincide with the expression given in eq. (9). These polynomials can be framed within the Appell family. Nevertheless, they deserve to be studied carefully and this will be done in a next paper.

\section{REFERENCES}

[1] D. Babusci, G. Dattoli, and M. Del Franco, Lectures on Mathematical Methods for Physics, Internal Report ENEA RT/2010/5837.

[2] P. Appell, J. Kampé de Fériét, Fonctions Hypergéometriqués et Polynômes d'Hermite, Gauthier-Villars, Paris (1926).

[3] D. Babusci, G. Dattoli, G. H. E. Duchamp, K. Górska, K. A. Penson, arXiv:1105.5967v1 [math.CA].

[4] H. M. Srivastava, H. L. Manocha, A Treatise on Generating Functions, Wiley, New York (1984).

[5] L. C. Andrews. Special functions for Engineers and Applied Mathematicians. Mac Millan, New York (1985).

infN - Laboratori Nazionali di Frascati, via E. Fermi, 40, IT 00044 Frascati (Roma), Italy

E-mail address: danilo.babusci@lnf.infn.it

ENEA - Centro Ricerche Frascati, via E. Fermi, 45, it 00044 Frascati (Roma), Italy

E-mail address: giuseppe.dattoli@enea.it

Dipartimento di Metodi e Modelli Matematici per le Scienze Applicate, Università degli Studi di Roma "Sapienza", via A. Scarpa, 14, Roma, Italy

E-mail address: germano@dmmm.uniroma1.it

Dipartimento di Metodi e Modelli Matematici per le Scienze Applicate, Università degli Studi di Roma "SApienzA", via A. Scarpa, 14, Roma, Italy

E-mail address: martinelli@dmmm.uniroma1.it

International Telematic University Uninettuno, Corso Vittorio Emanuele II, 39, 00186 Roma, ITALY

E-mail address: paoloemilioricci@gmail.com 